\title{
Einsatz eines Grundwasserströmungsmodells bei der Grundwassergewinnung im Xantener Stauchmoränenbogen (Niederrheinische Tiefebene)
}

\author{
Horst LOSEN \& Christoph TresKatis*) \\ Groundwater in a pushmoraine, groundwater flowsimulations, protection of drinking water, \\ drinkwater rewinning in unclaimed areas, Lower Rhine Embayment
}

\begin{abstract}
Kurzfassung: Die modernen Anforderungen an den Trinkwasserschutz, die zunehmende Zersiedelung und die landwirtschaftliche Nutzung weiter Teile der Niederrheinischen Bucht führen immer häufiger zu Nutzungskonflikten. Da die einmal vorhandenen Nutzungsstrukturen wie Naßabgrabungen und Gewerbegebiete die Flächen für eine schutzfähige Grundwassergewinnung reduzieren, bleibt oft nur die Verlagerung der Trinkwassergewinnung in zuvor nicht beanspruchte Gebiete.
\end{abstract}

Am Beispiel einer Prognose für die Verschiebung einer Entnahmegalerie aus einer Zone mit gewerblicher und lagerstättentechnischer Nutzung im Umfeld des Xantener Stauchmoränenbogens kann gezeigt werden, daß auf der Grundlage quartärgeologischer Grundlagendaten und eines numerischen Grundwasserströmungsmodells eine Optimierung der Ortslage für eine Trinkwassergewinnung durchgeführt werden kann.

Die eiszeitliche Überprägung weiter Teile des hydrogeologischen Grundwasserleitersystems im Xantener Bogen bedingte zahlreiche Restriktionen für die Optimierung eines Grundwassergewinnungsbereichs.

Mit Hilfe der Geländeaufnahmen und der Modellrechnungen wurden verschiedene neue Brunnenstandorte simuliert und hinsichtlich der ökologischen und hydrogeologischen Restriktionen bewertet.

Die Modellrechnungen ermöglichten bereits im Planungsstadium eine Vorausschau auf die Auswirkungen und den Nutzen einer Verlagerung der ursprünglichen Entnahmeschwerpunkte. Die Ergebnisse zeigen aber auch, daß ein Ausweichen der Trinkwassergewinnung zugunsten anderer Flächennutzungen mit neuen Restriktionen am neuen Standort verbunden sein kann und somit in jedem Einzelfall zu überprüfen ist.

\section{[Employment of a groundwater flow model in determining the position of production water wells in the pushmoraine arc of Xanten (Lower Rhine Basin, West Germany)]}

Abstract: Modern requirements for the protection of drinkingwater, the more increasing housing development and agricultural use of vast areas of the Lower Rhine Basin have

*) Anschriften der Verfasser: Dr. C. Treskatis, Ing.-Büro Bieske und Partner, Im Pesch 79, 53797 Lohmar 1; Dipl.Geol. Dr. H. LosEn; Wilhelmstraße 45, 50996 Köln 50 lead to conflicts of utilization. Because of existing structures of use, such as gravel excavations and commercial sites have reduced the areas which garantee a protected groundwater extraction, it has become inevitable for drinkingwater to be pumped in once still unclaimed areas.

Using the example of a prognosis for the displacement of an water well gallery out of the catchmentzone with commercial and raw material excavational use in the region of Xanten, it can be shown, that on the basis of quaternarie geological data and numeric groundwater flow simulations an optimization of a site for groundwater extraction can be determined.

The overimprinting of vast areas of the groundwater system of the pushmoraine arc near Xanten in the Pleistocene resulted in nomerous hydrogeological restrictions for the optimization of a water well gallery.

With help of field investigations and numeric calculations various new well positions were simulated and accessed in regard of ecological and hydrogeological restrictions.

The calculations allow already in the planing stage, for a prediction to be made on the effectivness and the feasibility of a displacement of the original water wells.

The results show however also that an evasion of groundwater extraction in favour of other areal usage, can be bound with new restrictions at the new location and must therefore be examined closely for each case.

\section{Einführung}

Der Xantener Stauchmoränenbogen liegt in einer der grundwasserreichsten Landschaften der Bundesrepublik Deutschland. Die Grundwasservorkommen sind hier an die ergiebigen kiesig-sandigen quartären Lockergesteine des niederrheinischen Tieflandes gebunden (s. Abb. 1).

Die Grundwassergewinnung erfolgt vorwiegend im Verbreitungsgebiet der quartären Terrassensedimente, deren Kies- und Sandvorkommen in der Rheinaue auch in zahlreichen Naßabgrabungen gewonnen werden.

Die konkurrierenden Ansprüche des Kiesabbaus sowie des Grundwasserschutzes treffen im Raum Xanten wie in vielen anderen, ähnlich strukturierten Ge- 


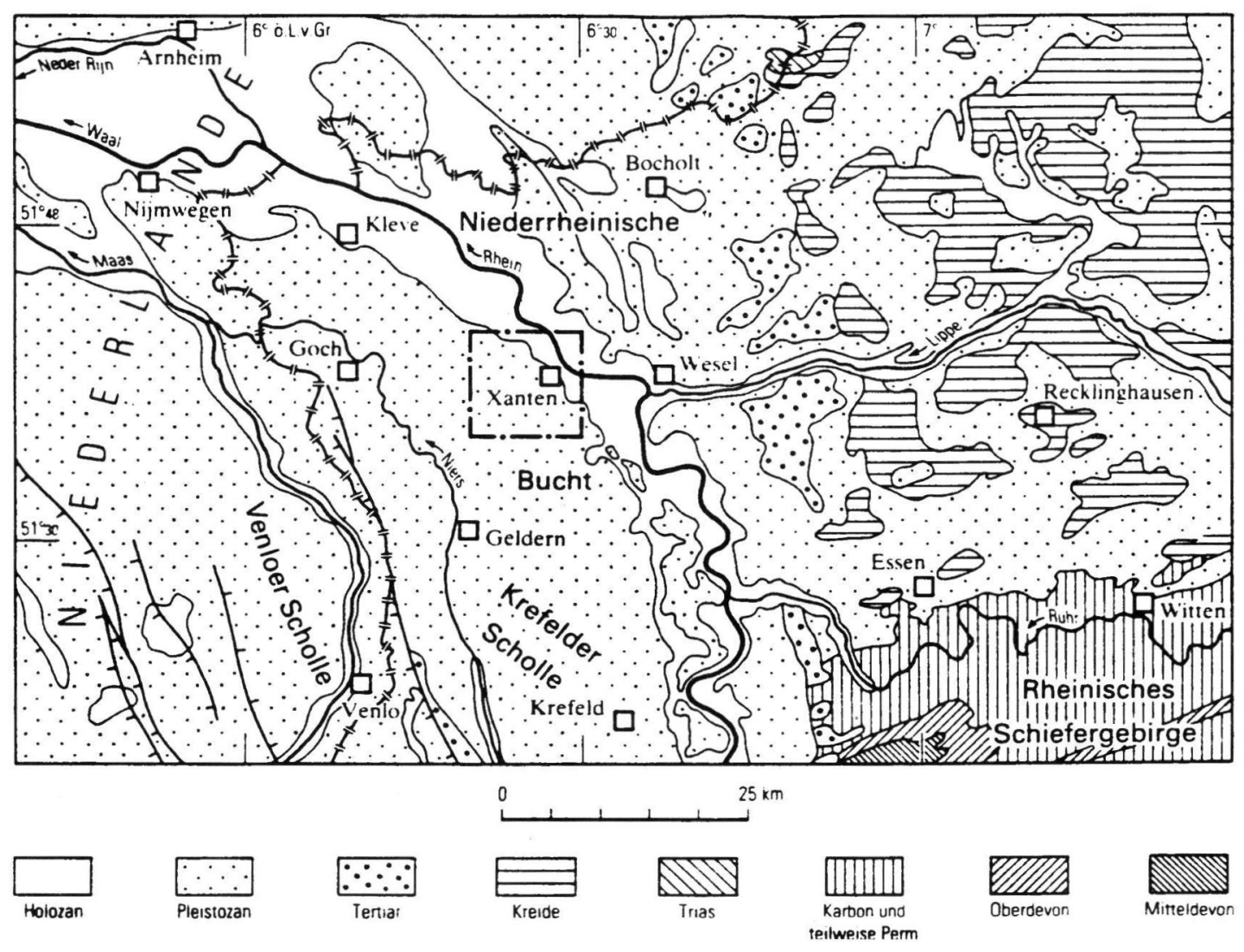

Abb. 1: Geographische Lage Xantens innerhalb des Niederrheingebietes. Aus Klostermann (1989a: 11).

bieten entlang des Rheins immer härter aufeinander. Zusätzlich entsteht durch die zunehmende Urbanisierung ehemals ländlicher Regionen und durch die Ausweisung von Gewerbe- und Industrieflächen ein weiterer Nutzungskonflikt, der die immer kleiner werdenden Flächen für eine Grundwassergewinnung weiter reduziert.

Wird der Schutz der weitflächigen Grundwasservorkommen im Rheintal durch städtebauliche Maßnahmen und voranschreitenden Lagerstättenabbau weiter bedroht, bleibt besonders für die Trinkwassergewinnung oft nur die Aufgabe der gefährdeten Brunnen.

Im Xantener Stauchmoränenbogen wurde mit Hilfe moderner hydrogeologischer und geologischer Erkundungsmethoden sowie durch detaillierte Modellrechnungen versucht, die Auswirkungen der möglichen Gefährdungspotentiale durch Verlagerung einer Grundwasserentnahmegalerie zu minimieren.

Die Verlagerung einer bisher hydraulisch einwandfrei funktionierenden Gewinnungsanlage stößt durch die "Neuerschließung" bisher ungenutzter und vielfach - im Sinne des Trinkwasserschutzes ungeschützter Grundwasserleiterbereiche oft an limitierende geologische Randbedingungen sowie an die Grenzen technischer und wirtschaftlicher Durchführbarkeit.

Im Verlauf der Untersuchungen zeigte sich, daß z. B. durch die Verlagerung der Teileinzugsgebiete der Brunnen Veränderungen im Rohwasserchemismus auftreten können.

Daneben werden durch die Verlagerung der förderbedingten Absenkungstrichter vorher unbeeinflußte Gebiete betroffen, in denen sich z. B. grundwasserabhängige Fließgewässer mit entsprechender Fauna und Flora entwickelt haben.

Vor diesem Hintergrund entstehen neue Anforderungen an die klassischen Methoden der Grundwassererschließung, die nur auf der Grundlage möglichst exakter quartärgeologischer Daten und Karten erfüllt werden können.

Durch hydrologische Modellrechnungen, die auf der Basis von Geländemessungen aus dem Xantener Stauchmoränenbogen aufgebaut wurden, können die verlagerungsbedingten Probleme erkannt und 


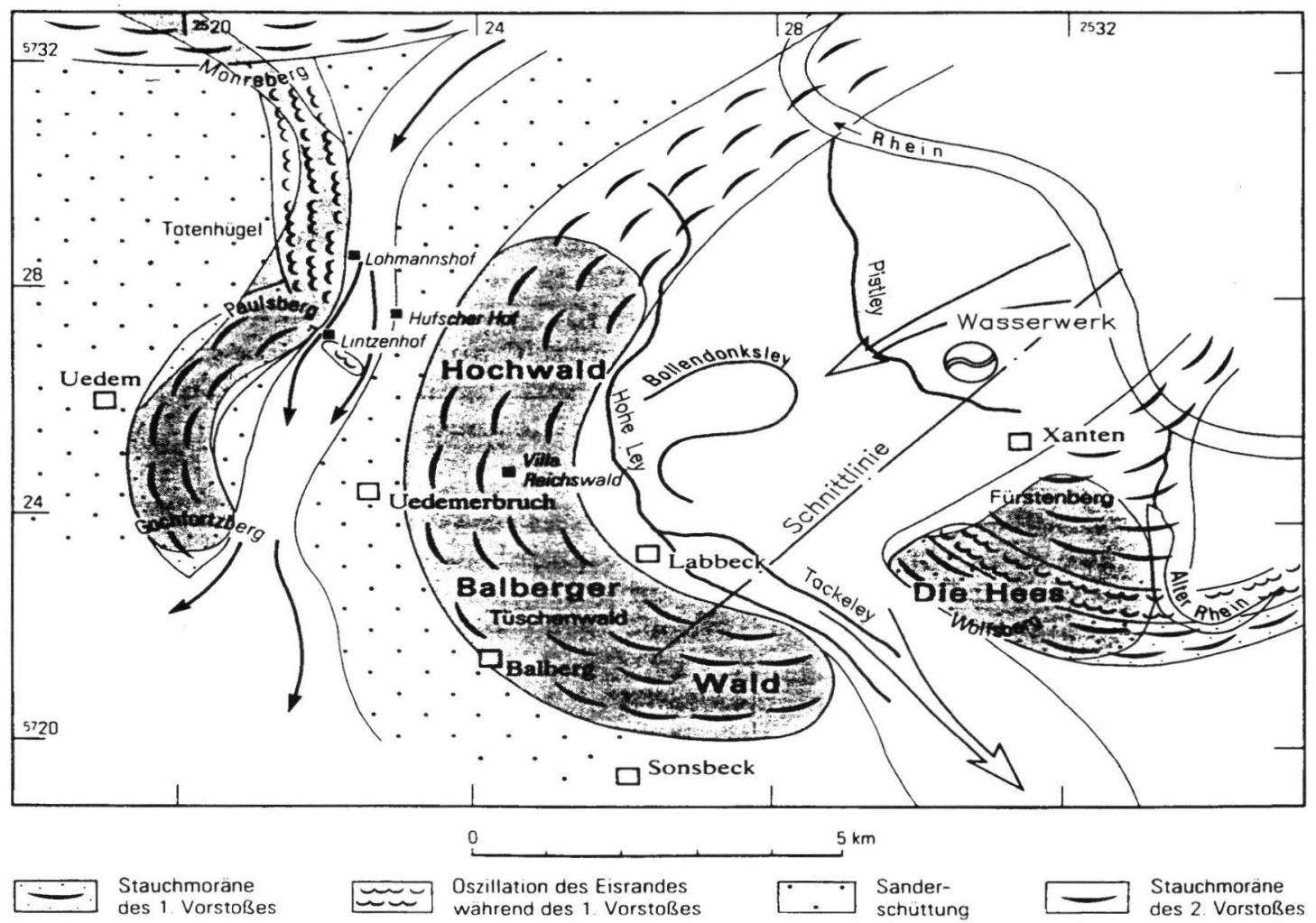

Abb. 2: Inlandeisvorstoß der Saale-Kaltzeit in der Umgebung von Xanten (letzte Phase).Aus KLostermann (1989a: 21).

deren Auswirkungen auf den Grundwasserhaushalt prognostiziert und quantifiziert werden.

Die letztendliche Beurteilung der Modellergebnisse darf aber nicht nur vor dem Hintergrund der technischen Durchführbarkeit gesehen werden, sondern muß auch Überlegungen über die langfristigen güteund wasserwirtschaftlichen Konsequenzen einer Aufgabe bisher genutzter Regionen zugunsten einer zunehmenden Flächenversiegelung beinhalten.

\section{Geologie und Hydrogeologie des Xantener Raumes}

\subsection{Lage und Morphologie}

Das Modellgebiet umfaßt den sogenannten Xantener Stauchmoränenbogen in der näheren Umgebung des Stadtgebietes Xanten. Im Osten bildet der Rhein und im Westen der Höhenzug des Hochwaldes und des Balberger Waldes die Gebietsgrenze (vgl. Abb. 2). Zwischen der Ortschaft Marienbaum und dem Rhein verläuft die nördliche Grenze des Modellgebietes, während im Süden der Rand zum Stauchrücken der Hees die Gebietsgrenze markiert. Der Balberger Wald, der Hochwald und die Hees gehören zum Bereich der Stauchmoränen, die sich als bogenförmiger Höhenzug mit Höhen von NN +70 bis $+85 \mathrm{~m}$ morphologisch deutlich von dem Ter- rassenbereich mit Geländehöhen um NN +22 m abheben. Das flachwellige Relief der Niederterrasse innerhalb des Moränenbogens wird gelegentlich von bis zu $8 \mathrm{~m}$ aufragenden Dünen unterbrochen

Der westliche Niederterrassenbereich wird von einem weit verzweigten Gewässersystem durchzogen, während zum Rhein hin Kiesabgrabungen und verlandete Altarme die Landschaft prägen.

\subsection{Geologischer Rahmen der Grundwasservorkommen}

Die Landschaftselemente des Modellgebietes bestehen aus känozoischen Ablagerungen, die zu verschiedenen Zeiten entstanden sind und ihre Entstehung jeweils anderen geologischen Vorgängen zu verdanken haben (KLOSTERMANN 1989 a).

Großräumig gesehen liegt das Untersuchungsgebiet im Niederrheinischen Tiefland, das ein Teilgebiet der Niederrheinischen Bucht ist.

In den seit dem frühen Oligozän sich absenkenden Niederrhein drang in mehreren Phasen die tertiärzeitliche Nordsee nach Süden vor und hinterließ einen mächtigen Sedimentstapel flachmariner Feinsedimente (s. Tab. 1). 


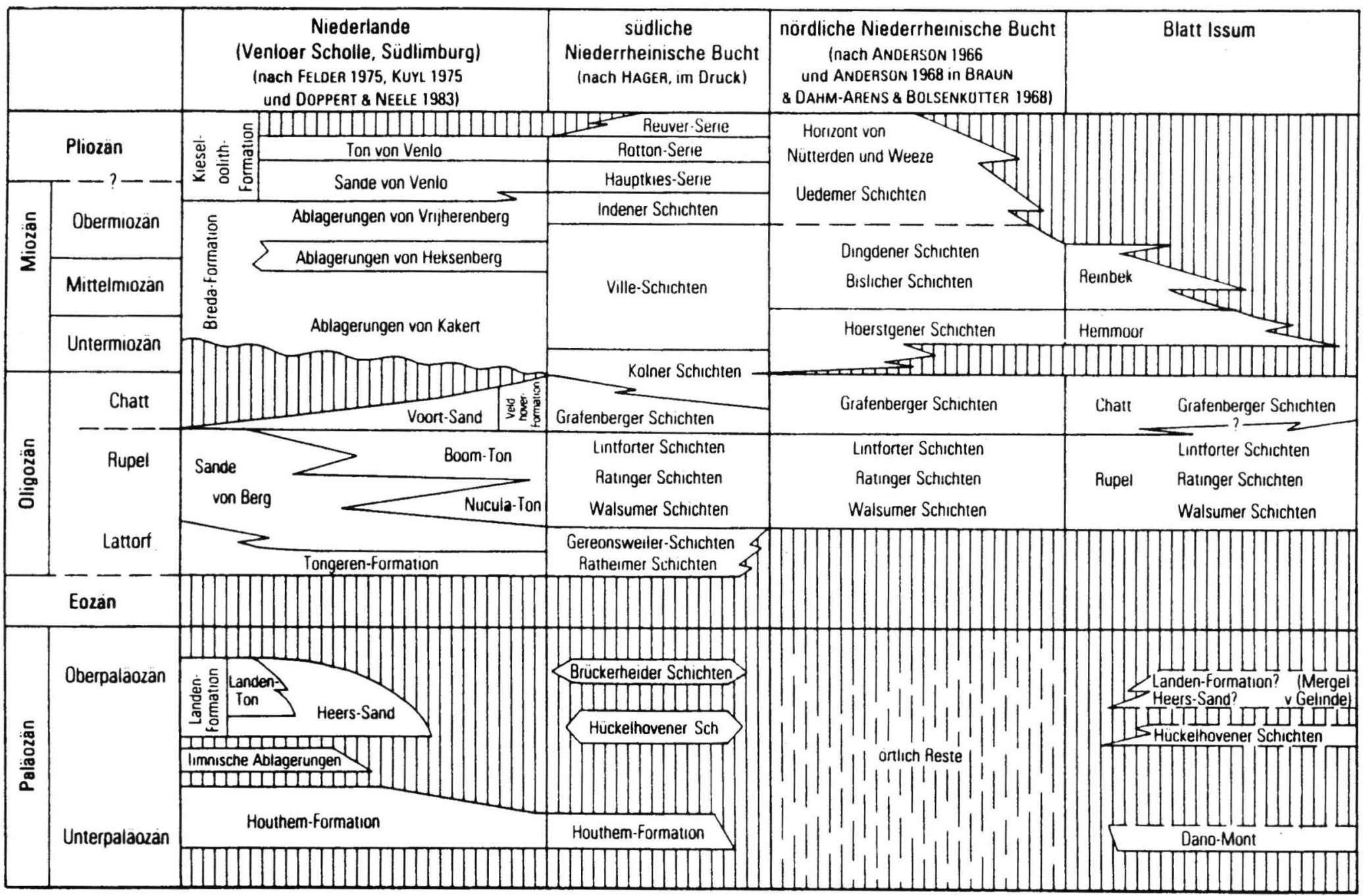


Die tertiären Meeresablagerungen reichen aufgrund glazialer Vorgänge des Eiszeitalters im Umfeld und innerhalb der Höhenrücken des Hochwaldes, Balberger Waldes, der Hees und des Fürstenberges bis nahe an die Geländeoberfläche heran (KLOSTERmanN
1989 a). Oberoligozäne Schichten des Chatt (Grafenberger Schichten) haben im tieferen Untergrund des Modellgebietes die größte Verbreitung. Sie bestehen aus einer Wechselfolge von grauen, schluffigfeinsandigen Tonen oder Schluffen mit graugrünen,

Tab. 2: Gliederung des Quartärs. Aus Klostermann (1989a: 15).

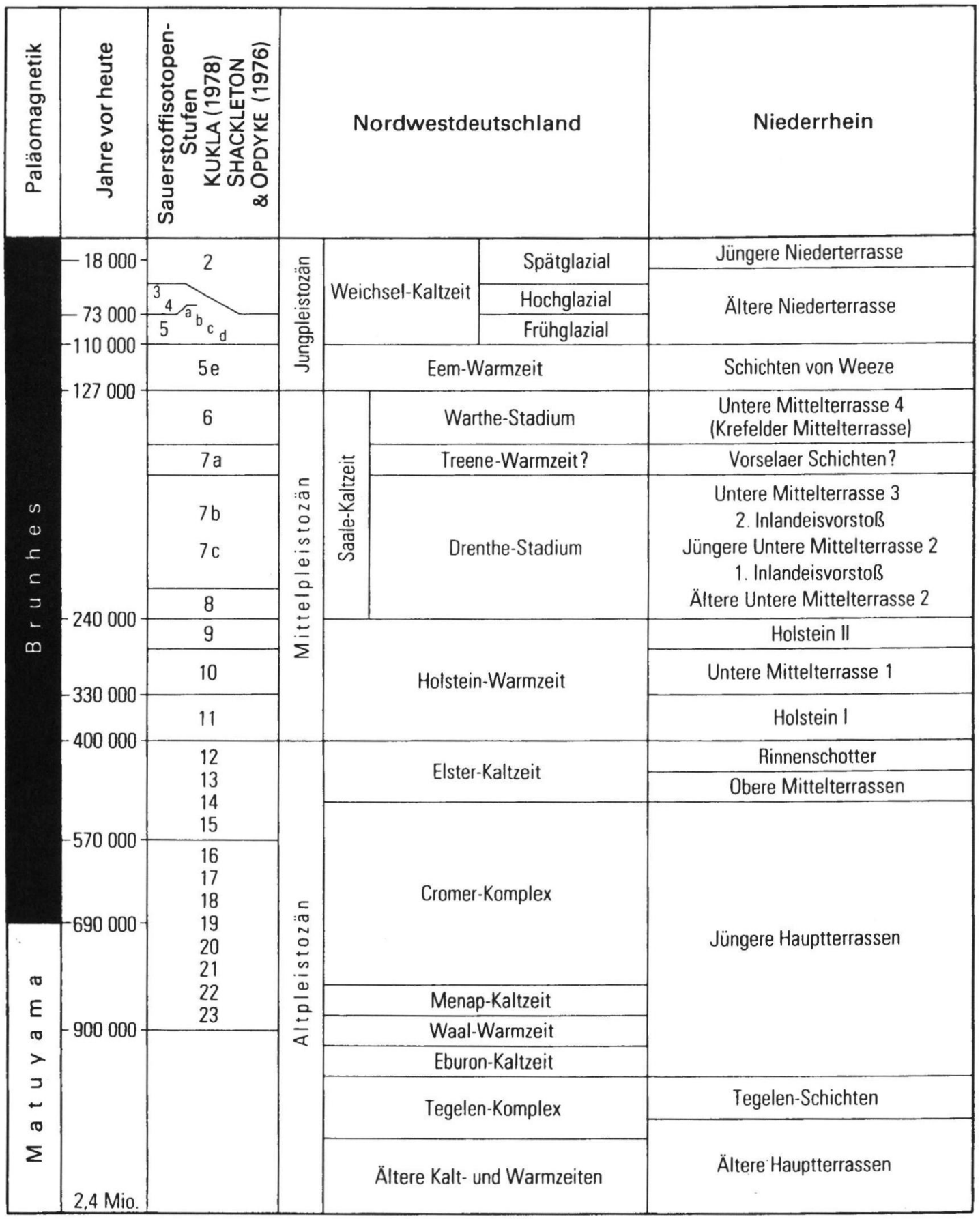


zum Teil schluffigen Feinsanden (KLOSTERMANn 1989 b). Darüber lagern miozäne und, im Norden und Nordwesten des Untersuchungsraumes, auch obermiozäne Feinsedimente.

Die oberflächennah anstehenden Gesteine des Xantener Stauchmoränenbogens bestehen aus pleistozänen und holozänen Ablagerungen (s. Tab. 2). Die Gesteinsausbildung reicht von lockeren Kiesen, Sanden, Schluffen und Tonen bis zu Torfen.

Die Stauchmoränenrücken des Xantener Bogens bestehen aus Kernstauchmoränen, die überwiegend während des zweiten saaleeiszeitlichen Eisvorstoßes aufgepreßt wurden (vgl. Abb. 2, KLOstermann 1990).

Sie bestehen aus einem festgelagerten Feinsandkern aus obermiozänen Sedimenten, die aufgrund von Druckunterschieden an der Gletscherzunge als hartgefrorene Platten entlang von Schwächezonen vor der Eisfront aufgestaucht und zusammengepreßt wurden.

Mit den obermiozänen Feinsanden wurden die ebenfalls gefrorenen pleistozänen Deckschichten hochgepreßt, so daß die Einhüllende der tertiären Feinsande und die heutige Erdoberfläche beziehungsweise die Oberfläche der kiesig-sandigen Terrassenablagerungen nahezu parallel verlaufen (Klostermann 1990).

Die quartäre Schichtenfolge erreicht in den vom Inlandeis nur wenig beanspruchten Zonen Mächtigkeiten von bis zu $25 \mathrm{~m}$, während in den gestauchten Gebieten durch Schichtverdopplung Mächtigkeiten bis zu $70 \mathrm{~m}$ auftreten können.

Im Pleistozän entwickelte sich im Xantener Bogen ein verschachteltes Flußsystem, dessen fluviatile Grobsedimente vereinzelt durch warmzeitliche Tone und Torfe des Eem-Interglazials untergliedert werden.

Im Modellgebiet lassen sich nach KLostermanN (1989 b) drei Terrassenkörper unterscheiden, die für die hydrogeologische Entwicklung von Bedeutung sind:

\section{- Untere Mittelterrasse 2 \\ - Niederterrasse \\ - Reihenterrassen 1 bis 4}

Die Untere Mittelterrasse 2 wird aus kiesig-sandigen Sedimenten aus der Saale-Kaltzeit mit Mächtigkeiten bis zu $20 \mathrm{~m}$ gebildet (vgl. Abb. 3). Diese Terrassenkörper lagern unmittelbar auf den tonig-schluffigen Beckenschluffen der Saale-Kaltzeit, die weitgehend durch die Einwirkungen der Gletschervorstöße überprüft wurden.

Innerhalb der Unteren Mittelterrasse 2 unterscheidet man einen älteren und einen jüngeren Terrassenkörper. Die ältere Untere Mittelterrasse 2 wurde vom saaleeiszeitlichen Inlandeisvorstoß ebenfalls überprägt, so daß Rinnenschotter und die HolsteinTone in gestörter und schuppenartiger Lagerung anzutreffen sind. Der jüngere Teil der Unteren Mittelterrasse 2 liegt ungestört vor.

In der folgenden Eem-Warmzeit wurde in dem nordwestlichen Teilbereich des Modellgebietes ein bis zu $2 \mathrm{~m}$ mächtiger Ton-/Torfhorizont und in der anschließenden Weichsel-Kaltzeit die bis zu $8 \mathrm{~m}$ mächtigen kiesig-sandigen Sedimente der Niederterrasse abgelagert.

Die jüngsten Terrassen des Rheins stammen aus dem Holozän. Dabei handelt es sich um die fluviatilen Grobsedimente der rund $10 \mathrm{~m}$ mächtigen Reihenterrassen des Rheins.

Die Reihenterrassen 1 bis 4 bildeten sich im Bereich der Niederterrasse des Rheins, die von Auensanden und -lehmen sowie Uferwallsedimenten überdeckt werden.

Äolische Sedimente und Torfe entstanden als junge Deckschichten auf den Stauchmoränen und in den flachen Talungen des Xantener Bogens. Am häufigsten treten Flugsande, Löß und Sandlöß im Umfeld der Stauchrücken auf. Dünen und Niedermoorablagerungen lassen sich in den ausgeprägten Mäanderschleifen der kleinen Fließgewässer zwischen Labbeck und Marienbaum nachweisen.

\subsection{Hydrogeologische Randbedingungen für die Grundwassergewinnung}

Die Terrassenlandschaft des Xantener Stauchmoränenbogens beherbergt ein grundwasserreiches Aquifersystem, das durch die saalekaltzeitlichen Überprägungen in drei Zonen gegliedert werden kann:

- Kernstauchmoränen mit gering wasserdurchlässigem Feinsandkern und einer Hülle gut bis sehr gut durchlässiger fluviatiler Ablagerungen.

- Pleistozäner Terrassenaquifer (Niederterrasse und Untere Mittelterrasse 2) des inneren Stauchmoränenbogens mit lokal eingelagertem Grundwasserstauer (Tone und Torfe des Eem).

- Holozäner Reihenterrassenaquifer der Rheinniederung mit unterlagernder Unteren Mittelterrasse 2.

Die Grundwasservorkommen in den Stauchmoränen des Xantener Bogens wurden aufgrund der komplexen Verschachtelung unterschiedlich gut wasserdurchlässiger Sedimente in der Vergangenheit kaum genutzt.

Die Erschließung dieser Vorkommen, wie z. B. im Norden des Hochwaldes bei Marienbaum, steht erst am Anfang (s. Klostermann \& Treskatis 1989 und TRESKATIS 1990). 


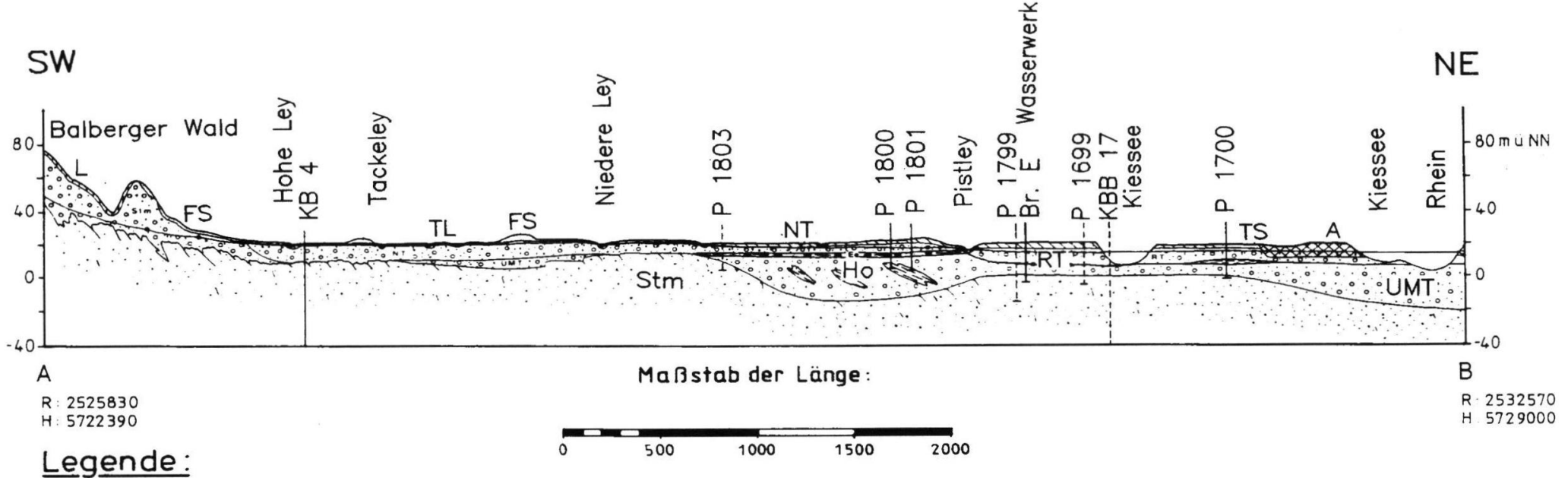

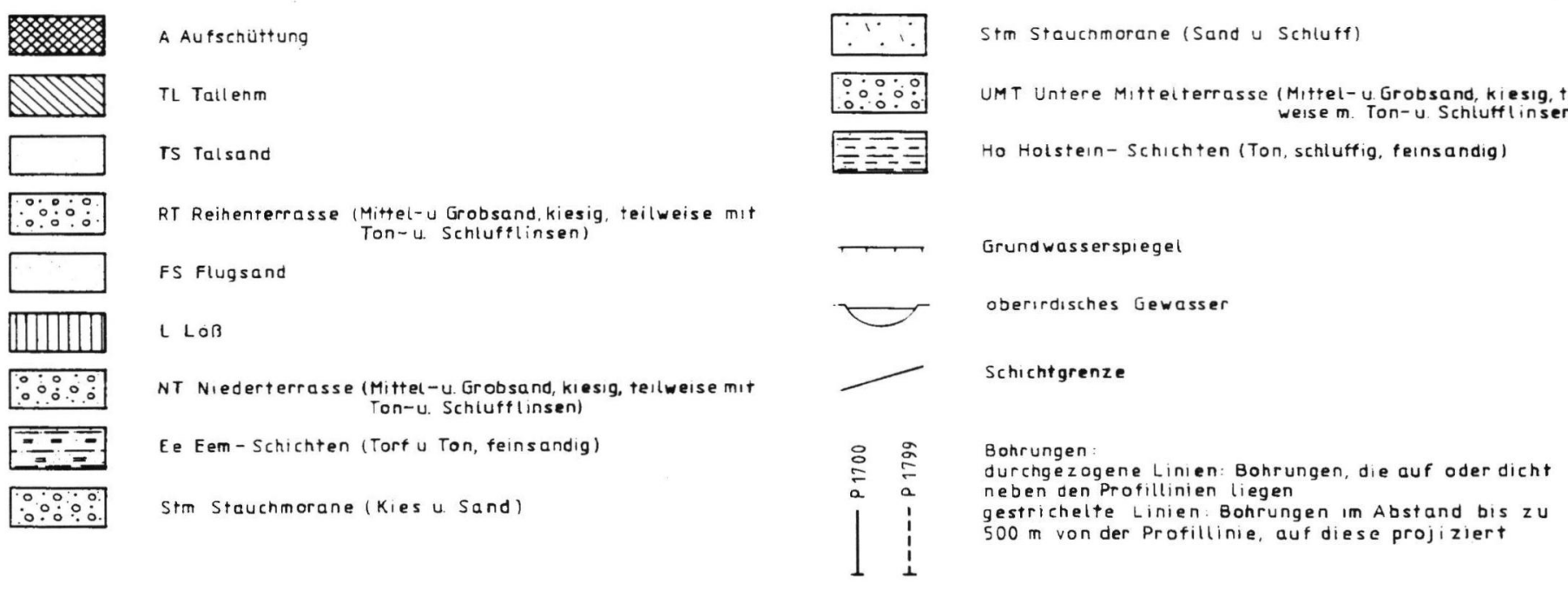


Eine Nutzung im Umfange der bisherigen Gewinnung von Trinkwasser aus den quartären Terrassensedimenten wird wegen der begrenzenden hydrogeologischen Randbedingungen in den Moränen nicht zu erwarten sein.

Im Inneren des Xantener Stauchmoränenbogens wird das lokal in einen gespannten und in einen freien Grundwasserleiter gegliederte obere Grundwasserstockwerk von den saalekaltzeitlichen Beckenschluffen unterlagert. Die geringere Durchlässigkeit der Beckenschluffe wirkt als hydraulische Barriere zwischen den sehr gut wasserdurchlässigen pleistozänen Flußsedimenten und den miozänen Flachmeerablagerungen im Liegenden der quartären Schichtenfolge (s. Abb. 3).

Der Grundwasserabfluß aus den morphologisch hervortretenden Stauchmoränen erfolgt im Inneren des Moränenbogens daher im wesentlichen über den pleistozänen Terrassenaquifer zur Rheinniederung. Ein ausgeprägtes Vorflutersystem nimmt bereits am Fuß der Höhenrücken einen Teil des unterirdischen Abflusses aus den Hüllschichten der Moränen auf.

Die glaziale Überprügung der Beckenschluffe sowie die geringen Durchflußquerschnitte der Terrassenkörper begünstigen dabei den Abtransport des Überschußwassers über Fließgewässer.

Die ergiebigsten Grundwasserleitergesteine des Modellgebietes schließen sich östlich der Pistley in der Rheinniederung an. Die bis zu mehr als $25 \mathrm{~m}$ mächtigen Schichten der holozänen Reihenterrassen und der Unteren Mittelterrasse zählen zu den wichtigsten Gebieten für die Grundwassergewinnung.

Die hohe Durchlässigkeit sowie die gegenüber dem Inneren des Stauchmoränenbogens mächtige Wassererfüllung der Terrassensedimente sorgen für einen optimalen Wasserandrang im Umfeld der Förderbrunnen. Die Einzugsgebiete der Brunnen werden häufig landwirtschaftlich intensiv genutzt oder werden von den anliegenden Kommunen städtebaulich verplant.

Bei der Ausweisung eines den modernen Anforderungen entsprechenden Trinkwasserschutzgebietes kann es für einen Wasserwerksbetreiber notwendig werden, nach Möglichkeiten zur Minimierung und Einstellung der Gefährdungspotentiale zu suchen.

Dabei kann auch die Verlagerung des Entnahmeschwerpunktes einer Brunnengalerie in Betracht gezogen werden, falls die örtlichen Gegebenheiten, wie z. B. Naßabgrabungen oder ausgewiesene Gewerbeflächen, mit den Ausweisungskriterien für die Festlegung einer Trinkwasserschutzzone kollidieren.

\section{Grundwasserströmungsmodell}

Für die zuverlässige Prognostizierung der Auswirkungen einer Verlagerung von Förderbrunnen auf den Grundwasserstand und auf das Abflußverhalten der im Modellgebiet gelegenen Vorfluter wurde für den Xantener Stauchmoränenbogen ein instationäres horizontal-ebenes Grundwasserströmungsmodell installiert.

\subsection{Modellaufbau und Randbedingungen}

Das Modellgebiet ist durch ein quadratisches Rasternetz mit 200 m Knotenabstand und insgesamt 1113 Knotenpunkten in diskrete Teilbereiche eingeteilt (vgl. Abb. 4). Vor der Diskretisierung und der Kalibrierung des Modells müssen anhand des verfügbaren Datenmaterials der hydrogeologische Aufbau des Modells und die Randbedingungen ermittelt werden. Die wichtigsten Randbedingungen sind:

- Aquiferaufbau und Aquifersohle

- Grundwasserentnahmen

- Grundwasserströmung

- Grundwasserneubildung

- Vorflutereinfluß auf das Grundwasser

- Durchlässigkeit im Aquifer

- Modellränder.

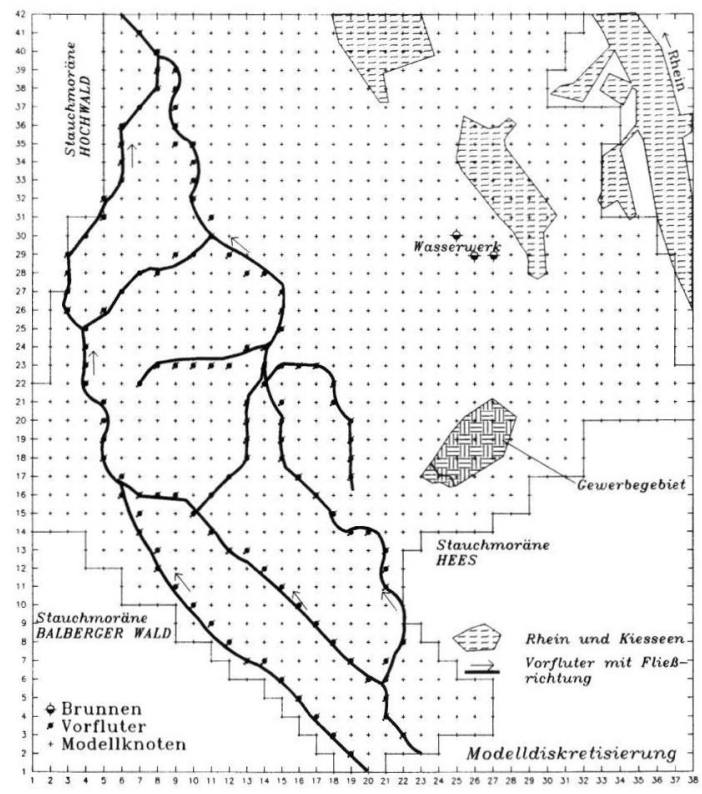

Abb. 4: Modelldiskretisierung

Die Installation von Grundwasserströmungsmodellen bedingt eine intensive Erkundung der hydrogeologischen und wasserwirtschaftlichen Verhältnisse im Modellgebiet und dessen näherer Umge- 
bung. Der Umfang und die Qualität der System- und Randbedingungen bestimmen die Genauigkeit der Kalibrierung und der Prognoserechnungen. Für die Diskretisierung des vorliegenden Grundwasserströmungsmodells mußten die bestehenden hydrogeologischen Daten durch umfangreiche Feldarbeiten (Einrichtung von zahlreichen neuen Grundwassermeßstellen zur Grundwasserstandsmessung, Pumpversuche, Messung des Vorfluterabflusses in Abhängigkeit des Grundwasserstandes, Ermittlung der Sohlendurchlässigkeit der Vorfluter und Leakageraten der Eem-Schichten) ergänzt werden.

\subsubsection{Aquiferaufbau und Aquifersohle}

Innerhalb des Modellgebietes stellen die überwiegend kiesig-sandigen Sedimente der Reihen-, Nieder- und Unteren Mittelterrasse den Hauptaquifer dar (vgl. Abb. 3). Die Durchlässigkeiten des Aquifers liegen nach Pumpversuchsergebnissen im Mittel bei $2 \cdot 10^{-3} \mathrm{~m} / \mathrm{s}$ und stimmen gut mit den berechneten modellwirksamen Durchlässigkeiten überein. In den Sedimenten der Unteren Mittelterrasse sind schuppenartige Strukturen der tonig-schluffigen Holstein-Schichten eingelagert mit Durchlässigkeiten zwischen $10^{-6}$ und $10^{-9} \mathrm{~m} / \mathrm{s}$.

Im Nordosten des Modellgebietes ist zwischen der Unteren Mittelterrasse und der Niederterrasse eine 0,8 bis $2 \mathrm{~m}$ mächtige Torf- und Tonschicht der EemWarmzeit mit Durchlässigkeiten kleiner als $1 \cdot 10^{-6}$ $\mathrm{m} / \mathrm{s}$ eingelagert. Aufgrund der geringen Durchlässigkeit der Eem-Sedimente liegt hier ein partieller Grundwasserstockwerksbau mit gespannten Grundwasserverhältnissen im Bereich der Unteren Mittelterrasse vor.

Die Aquifersohle wird von den eiszeitlich überprägten schluffig-feinsandigen Beckenchluffen gebildet. Nach Klostermann (1989 b) liegt die Durchlässigkeit bei $4 \cdot 10^{-5} \mathrm{~m} / \mathrm{s}$. Die Oberfläche der Aquiferbasis im Modellgebiet zeigt ein bewegtes Relief mit übertieften Rinnenstrukturen sowie Tiefenlagen von NN -20 $m$ und Hochbereichen mit Höhenlagen zwischen $\mathrm{NN}+10$ und $+15 \mathrm{~m}$. Dementsprechend schwankt die grundwassererfüllte Mächtigkeit des Hauptaquifers zwischen 4 und $42 \mathrm{~m}$.

\subsubsection{Grundwasserentnahmen und Grundwasserströmung}

Die Grundwasserentnahmen für die öffentliche Trinkwasserversorgung erfolgt mittels Grundwasserförderbrunnen aus dem Bereich der holozänen Reihenterrassen. Das Einzugsgebiet dieser Brunnen liegt im Südwesten und Süden des Modellgebietes (vgl. Abb. 5).

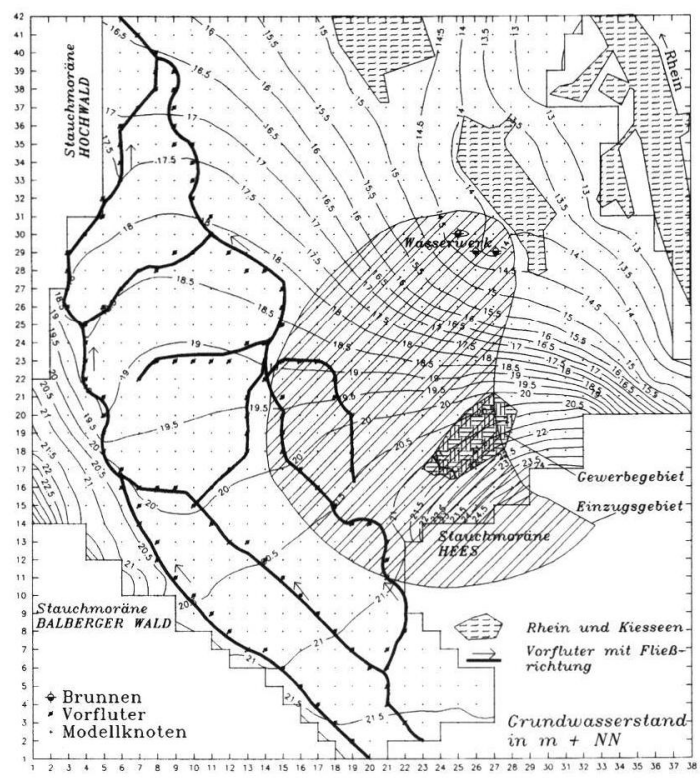

Abb. 5: Mittlerer Bezugswasserspiegel im quartären Grundwasserleiter

Das Grundwasserströmungsbild im Modellgebiet weist nach Grundwasserganglinienanalysen durchschnittlich an mehr als 300 Tagen im Jahr weitgehend stationäre Grundwasserströmungsverhältnisse auf. Zwischen Rhein und Hohe Ley ist eine Grundwasserscheide ausgebildet, die sich nur bei Hochwasserereignissen im Rhein kurzfristig geringfügig verlagern kann. In Abbildung 5 ist ein repräsentativer mittlerer Grundwasserstand dargestellt, der die Abstromverhältnisse im Stauchmoränenbogen und in den angrenzenden Terrassenkörpern verdeutlicht.

\subsubsection{Grundwasserneubildung und Wasserhaushalt}

Die Grundwasserneubildung ist die am schwierigsten zu bestimmende Randbedingung für das Grundwassermodell. Für die Modellrechnung werden die mittlere langfristige (für die Prognoserechnungen) und die modellrelevante Grundwasserneubildungsrate für den Kalibrierungszeitraum im Sommerhalbjahr 1990 benötigt.

Die langfristige Grundwasserneubildung für das Modellgebiet wurde mittels der Wasserhaushaltsgleichung berechnet:

$$
\mathrm{A}_{\mathrm{u}}=\mathrm{N}-\mathrm{V}-\mathrm{A}_{0}
$$

mit

$\mathrm{A}_{\mathrm{u}}=$ Grundwasserneubildung $(\mathrm{mm} / \mathrm{a})$;

$\mathrm{N}=$ Niederschlag $(\mathrm{mm} / \mathrm{a})$;

$\mathrm{V}=$ Verdunstung $(\mathrm{mm} / \mathrm{a})$;

$\mathrm{A}_{0}=$ oberirdischer Abfluß (mm/a). 
Speicheränderungen im Grundwasserraum bleiben dabei unberücksichtigt, da sie sich über längere Zeiträume ausgleichen. Für das Modellgebiet konnte eine mittlere langfristige Grundwasserneubildungsrate von $229 \mathrm{~mm} / \mathrm{a}\left(\hat{=} 7,3 \mathrm{l} /\left[\mathrm{s} \cdot \mathrm{km}^{2}\right]\right)$ berechnet werden. Die kurzfristige Grundwasserneubildungsrate für den Kalibrierungszeitraum im Frühjahr/Sommer 1990 wurde anhand einer Vergleichsrechnung zwischen dem tatsächlichen Niederschlag und den Kennwerten der mittleren monatlichen Verdunstungsraten (KELLER 1978; MinISTER FÜr UMWELT, RAUMORDNUNG UND LANDWIRTSCHAFT NW 1989 und ERFTVERBAND 1983) errechnet.

\subsubsection{Vorflutereinfluß auf das Grundwasser}

Zur Beurteilung des Einflusses der kleineren Vorfluter auf das Grundwasser sind im Modellgebiet die Hohe und Niedere Ley, die Tacke Ley und der Unterlauf der Willichsche Ley diskretisiert worden. Der Wasseraustausch zwischen Oberflächengewässer und Grundwasser wird nach dem Leaky-AquiferPrinzip ermittelt:

$$
\mathrm{A}_{\mathrm{L}}=\mathrm{F} \cdot \mathrm{k} \cdot \mathrm{h} / \mathrm{m}
$$

Darin bedeuten:

$$
\begin{aligned}
A_{\perp}= & \text { Aus- bzw. Zusickerungsrate des Vorfluters } \\
& \left(\mathrm{m}^{3} / \mathrm{s}\right) ; \\
\mathrm{F}= & \text { Fläche der Vorflutersohle }\left(\mathrm{m}^{2}\right) ; \\
\mathrm{k}= & \text { Durchlässigkeit der Vorflutersohle }(\mathrm{m} / \mathrm{s}) ; \\
\mathrm{h}= & \text { Potentialdifferenz zwischen Wasserstand im } \\
& \text { Vorfluter und Grundwasserstand }(\mathrm{m}) \\
\mathrm{m}= & \text { Mächtigkeit der Vorflutersohle }(\mathrm{m}) .
\end{aligned}
$$

Die o. g. Formel gilt nur für gesättigte Bereiche. Für die Ermittlung der Leakageraten zwischen Vorfluter und Grundwasser standen zahlreiche Abflußmessungen und Untersuchungen der Durchlässigkeit einzelner Bachsohlenbereiche zur Verfügung. Die Potentialhöhen zwischen Grundwasserstand und Wasserstand im Vorfluter wurden durch parallel zu den Grundwasserstandsmessungen durchgeführten Nivellements der Vorfluter ermittelt.

\subsubsection{Modellränder}

Bei der Diskretisierung der Modellränder wurden Bereiche mit Festspotentialen und Bereiche mit festen Ein-/Abstrommengen abgegrenzt. Bedingt durch instationäre Grundwasserströmungsverhältnisse am Rhein und im Übergangsbereich zwischen Stauchmoräne und Terrassenbereich wurden diese Modellränder mit Festpotentialen belegt. Die restlichen Modellränder wurden weitgehend als Ein-/Abstromrand definiert.

\section{Kalibrierung des Grundwasser- strömungsmodells}

Ziel der Kalibrierung des Grundwassermodells ist die Anpassung der Modellparameter an die hydrogeologischen Gegebenheiten. Zur Gewährleistung einer möglichst genauen Kalibrierung wurde das Grundwassermodell deshalb anhand von 5 Grundwasserständen im Frühjahr/Herbst 1990 kalibriert. Für jeden dieser Zeitschritte wurden die Randbedingungen modellunabhängig berechnet und für die Modellrechnung diskretisiert. Anschließend wurden die Durchlässigkeiten und das speichernutzbare Porenvolumen in einem instationären und halbautomatischen Rechenverfahren für sämtliche Zeitschritte optimiert. Innerhalb der möglichen Fehlergrenzen wurden die Randbedingungen variiert, um die Sensitivität des Modells zu testen.

\subsection{Ergebnisse der Modellkalibrierung}

Ein Maß für die Qualität der Kalibrierung unter Berücksichtigung der Beschaffenheit der Randbedingungen stellt der Vergleich zwischen berechneten und gemessenen Grundwasserständen dar. Liegen dabei die Abweichungen zwischen gemessenen und berechneten Grundwasserständen über sämtliche Zeitabschnitte innerhalb der zu erwartenden Fehlergrenzen, so ist die Kalibrierung erfolgreich abgeschlossen. In der Abbildung 6 sind die gemessenen und die berechneten Grundwasserstände für den 16. Mai 1990 beispielhaft dargestellt. Es zeigt sich, daß insgesamt eine gute Übereinstimmung bei der Kalibrierung erzielt werden konnte. Im Bereich der kleinen landseitigen Vorfluter liegt die durchschnittliche Abweichung zwischen gemessenen und berechneten Grundwasserständen im Durchschnitt bei rund $1,5 \mathrm{dm}$.

Für die Beurteilung der zeitlichen Entwicklung der Grundwasserstände sind in der Abbildung 7 die gemessenen und berechneten Grundwasserganglinien der im Modellgebiet gelegenen Grundwassermeßstellen 1801, 200222 und 200726 dargestellt. Zwischen gemessenen und berechneten Grundwasserständen ist eine gute Übereinstimmung zu erkennen.

\section{Berechnung der Fallbeispiele}

Nach der intensiven hydrogeologischen Erkundung des Modellgebietes und nach dem erfolgreichen $\mathrm{Ab}-$ schluß der Modellkalibrierung können mit dem Grundwasserströmungsmodell Fallbeispiele für eine mögliche Verlagerung der Brunnengalerie durchgeführt werden. Für eine Optimierung der Brunnenstandorte mittels des Grundwasserströmungsmodells waren folgende Restriktionen zu beachten: 
1. Das südlich der heutigen Brunnenstandorte gelegene Gewerbegebiet und die in Richtung Rhein gelegenen Kiesseen müssen außerhalb des Einzugsgebietes der zu verlagernden Trinkwasserbrunnenstandorte liegen (vgl. Abb. 5).

2. Die Wasserführung der im Xantener Stauchmoränenbogen gelegenen kleinen Vorfluter darf durch die Verlagerung der Brunnenstandorte nicht negativ beeinflußt werden.
Langfristige Grundwasserganglinienanalysen zeigen, daß die Grundwasserstände vom Sommer 1990 als niedrig einzustufen waren. Für die Beurteilung der Auswirkung der Verlagerung der Brunnen auf den Grundwasserstand wurde aufgrund fehlender Grundwasserstandsdaten aus der Vergangenheit für das gesamte Modellgebiet zunächst ein Bezugswasserspiegel (Abb. 5) berechnet, der einen mittleren repräsentativen Grundwasserstand darstellt. Anschließend wurden verschiedene Fallbeispiele mit

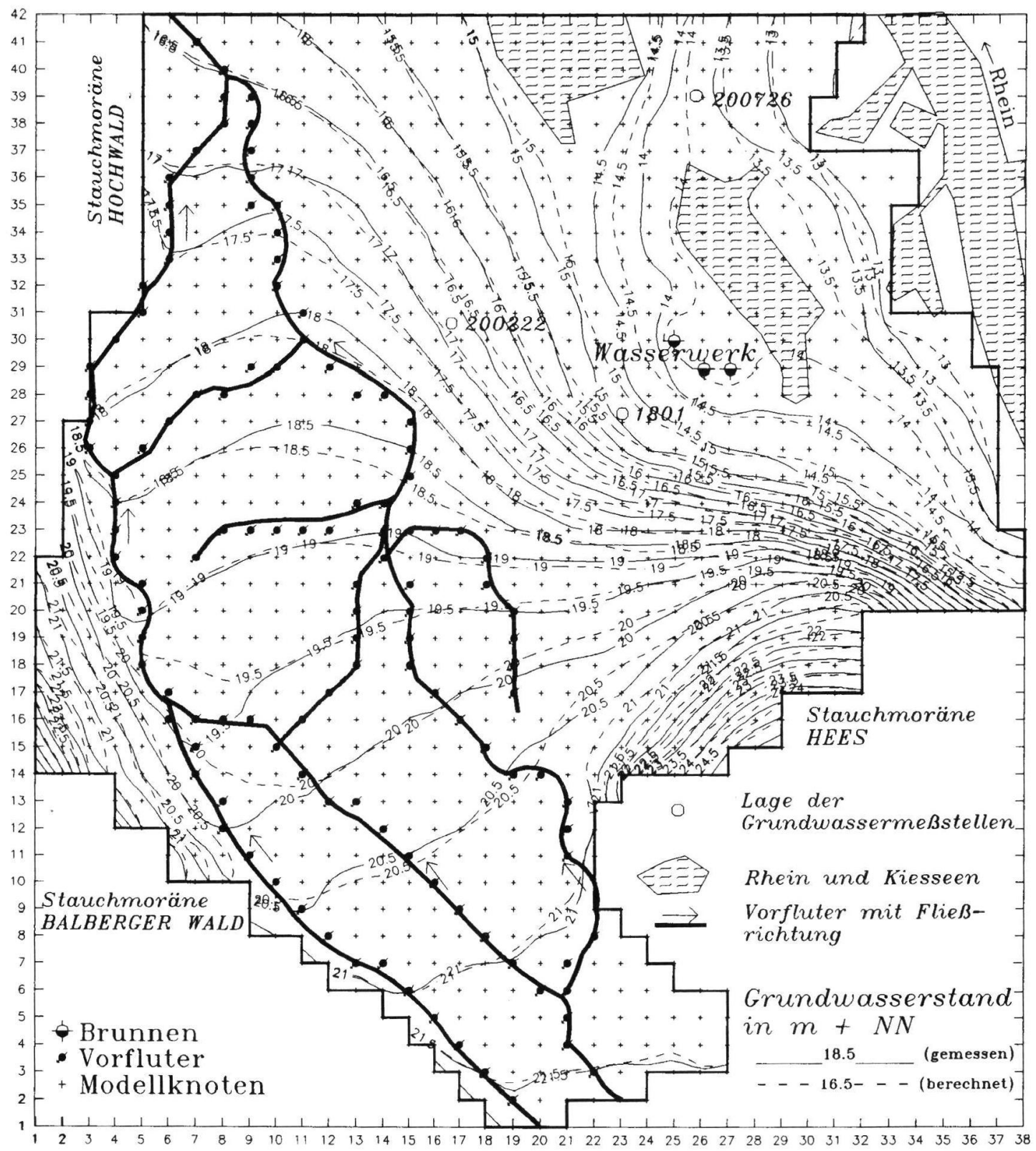

Abb. 6: Kalibrierung des Grundwasserstandes für den Zeitpunkt 16.5.90 
unterschiedlichen Ortslagen der Brunnen und Entnahmemengen berechnet und deren Auswirkungen gegenüber dem Bezugswasserstand analysiert. Allgemein wurden für sämtliche Fallbeispiele dabei die Randbedingungen aus den Ergebnissen der Kalibrierungsrechnungen (vgl. Abschnitt 3 und 4) übernommen. Berechnet wurden die hydraulischen Auswirkungen der zu verlagernden Trinkwasserbrunnen über einen Zeitraum von einem Jahr bei einer mittleren Grundwasserneubildungsrate von 229 $\mathrm{mm} / \mathrm{a}$.

\subsection{Bezugswasserspiegel}

Als Bezugswasserspiegel für die Beurteilung der Fallbeispiele wurden mit den Randbedingungen vom 16. Mai 1990 und der mittleren langjährigen Grundwasserneubildung von $229 \mathrm{~mm} / \mathrm{a}$ die Grundwasserstandsentwicklung für 365 Tage berechnet. Die Entnahmemenge aus den bestehenden Förderbrunnen wurde mit 2,7 Mio. $\mathrm{m}^{3} / \mathrm{a}$ eingesetzt. Ein Vergleich des Bezugswasserspiegels mit den Grundwasserständen und Wasserbilanzen aus dem Sommer 1990 zeigt einen um rund $1 \mathrm{~m}$ höheren Grundwasserstand für den Bezugswasserspiegel. Die Aussickerungsraten aus dem Aquifer in den Vorfluter liegen bei dem berechneten Bezugswasserspiegel gegenüber den Grundwasserständen vom Frühjahr 1990 um rund $25 \%$ höher.

\subsection{Fallbeispiele}

In der Nähe der heutigen Brunnenstandorte und im Südwesten des Modellgebietes zwischen Hohe Ley und Tacke Ley wurden insgesamt 4 mögliche Standorte unter Berücksichtigung der o. g. Restriktionen mit dem Grundwassermodell untersucht.

Brunnenstandorte im Bereich des südwestlichen Modellgebietes würden innerhalb des Stauchmoränenbogens eine Abflußminderung in den Vorflutern Tacke Ley und Hohe Ley ergeben. Zusammen mit den Kosten für die Verlagerung des gesamten Wasserwerkes erscheinen diese vorfluternahen Standorte nur bedingt für die Verlagerung der Förderbrunnen geeignet.

Als wasserwirtschaftlich und ökologisch günstigster Standort für die neuen Trinkwasserbrunnen konnte ein Bereich rund $500 \mathrm{~m}$ nordwestlich der bestehenden Brunnenanlage ermittelt werden. An den geplanten 3 neuen Brunnen wurde eine Grundwasserentnahme von 1,5 Mio. $\mathrm{m}^{3} / \mathrm{a}$ und an den alten Brunnen eine Entnahme von 1 Mio. $\mathrm{m}^{3} / \mathrm{a}$ simuliert. Die Ergebnisse der Simulationsrechnungen sind in dem Grundwassergleichenplan der Abbildung 8 dargestellt. Das Einzugsgebiet der neuen Brunnen verlagert sich um rund $200 \mathrm{~m}$ nach Osten, wobei die derzeitigen Kiesseen bei stationären Grundwasserströ-

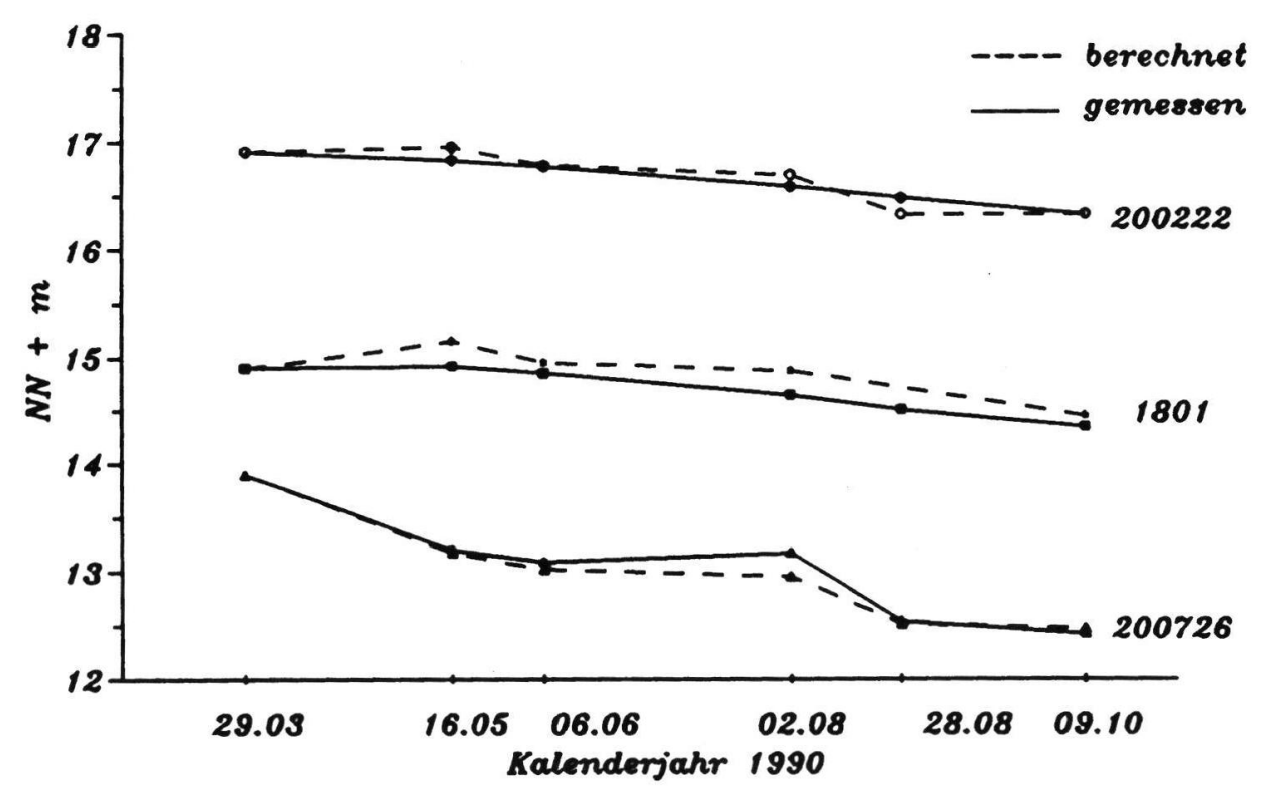

Abb. 7: Berechnete und gemessene Grundwasserganglinien der Eichmeßstellen (Ortslagen der Grundwassermeßstellen in Abb. 7) 
mungsverhältnissen außerhalb des Einzugsgebietes der neuen Brunnen liegen. Das bereits erwähnte Gewerbegebiet würde außerhalb des Einzugsgebietes der neuen Brunnen liegen. Die Leakageraten an den landseitigen Vorflutern bleiben von dieser Veränderung der Brunnenstandorte unbeeinflußt.

\section{Zusammenfassung}

Grundwasserströmungsmodelle eignen sich gut für die Optimierung von Standorten für Trinkwasserbrunnen hinsichtlich ökologischer und hydrogeologischer Restriktionen. Voraussetzung für den erfolgreichen Einsatz von Grundwasserströmungsmodellen ist eine umfassende Erkundung der hydrogeolo- gischen und wasserwirtschaftlichen Verhältnisse im Modellgebiet und dessen näherer Umgebung. Nach erfolgreicher Kalibrierung des Modells lassen sich anschließend vertrauenswürdige Prognoserechnungen mit unterschiedlichen Randbedingungen durchführen.

Im Einzugsgebiet einer Grundwassergewinnungsanlage im Bereich des Xantener Stauchmoränenbogens wurde ein instationäres Grundwasserströmungsmodell mit 1113 Modellknoten und $200 \mathrm{~m}$ Knotenabstand installiert. Anhand zahlreicher Feldmessungen und deren Auswertungsergebnisse wurden die modellrelevanten Beeinflussungsgrößen (wie z. B. Aquiferaufbau und -durchlässigkeit, Abhängigkeit des Vorfluterabflusses vom Grundwasserstand, Auswirkungen von rheinnahen Auskie-

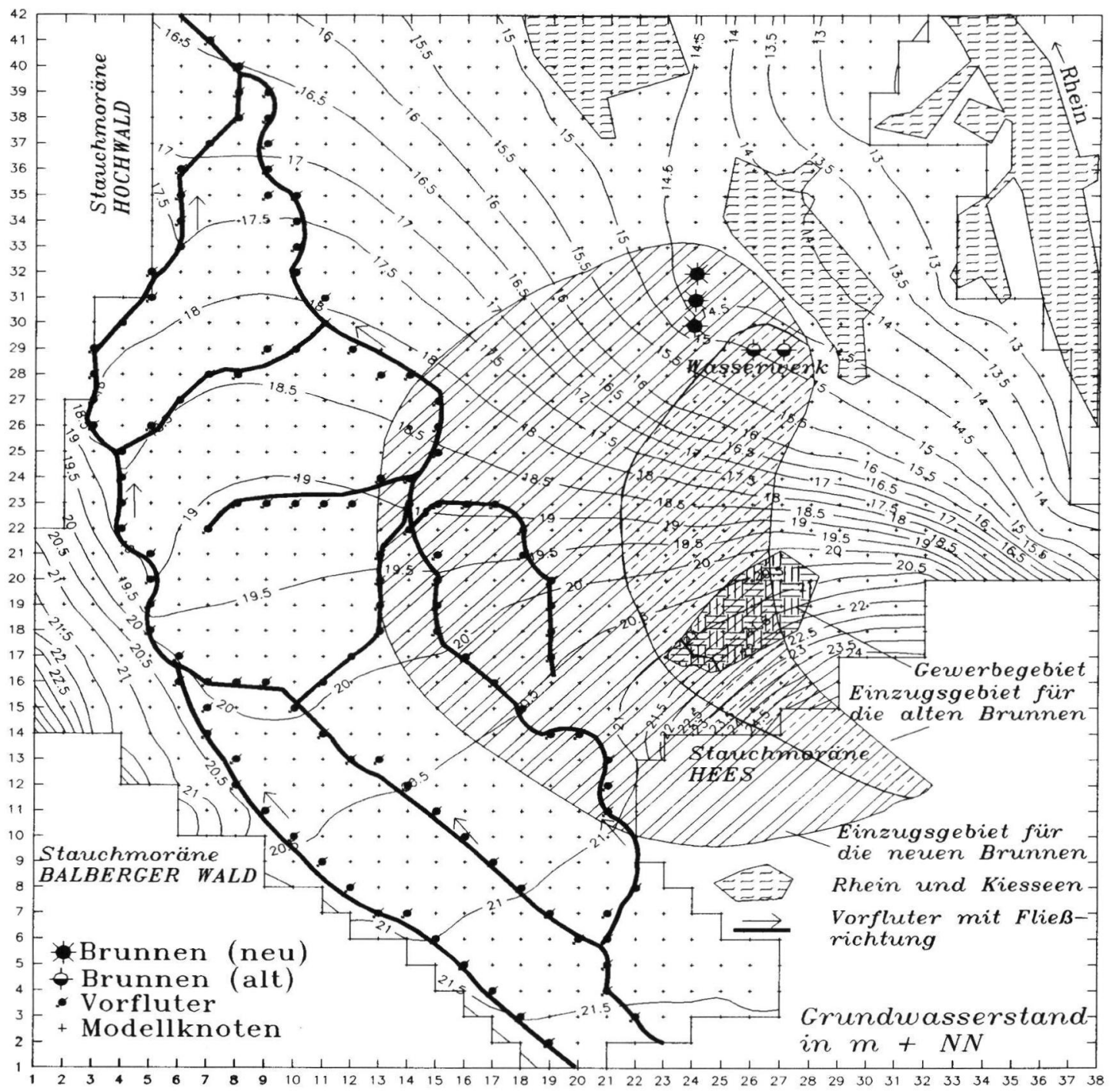

Abb. 8: Gerechneter Grundwasserstand für neue und alte Brunnenstandorte 
sungsbetrieben und langfristige Grundwasserstandsentwicklung) analysiert und für das Modell kalibriert.

Anschließend wurden 4 Fallbeispiele für unterschiedliche Brunnenstandorte und Entnahmemengen berechnet und mit einem simulierten repräsentativen Bezugswasserstand verglichen. Als optimaler Standort für die neuen Trinkwasserbrunnen konnte ein Bereich rund $500 \mathrm{~m}$ nördlich der bestehenden Brunnenanlagen ermittelt werden, der sämtliche Restriktionen hinsichtlich Grundwasserschutz und Ökologie erfüllt.

\section{Schriftenverzeichnis}

ERFTVERBAND (1983): Wasserversorgung im Verbandsgebiet. - 191 S., 99 Abb.; Bergheim.

KelleR, R. (1978): Hydrologischer Atlas der Bundesrepublik Deutschland. - Deutsche Forschungsgemeinschaft: 28 S., 71 Karten; Boppard (Boldt).

Klostermann, J. (1989 a): Die Entstehungsgeschichte der Xantener Landschaft. - Spurenlese - Beiträge zur Geschichte des Xantener Raums: S. 11 - 36, 10 Abb., 3 Tab., 4 Taf.; Köln (Rheinland-Verlag).
- (1989 b): Erläuterungen zu Blatt 4304 Xanten. - Geol. Karte von Nordrhein-Westfalen 1:25 000: 153 S., 14 Abb., 13 Tab., 3 Taf.; Krefeld (Geologisches Landesamt).

- (1990): Saalezeitliche Stauchmoränentypen am Niederrhein und ihre Entstehung. - N. Jb. Geol. Paläont. Abh. 181 (1 - 3): S. 455 - 470, 7 Abb., 1 Tab.; Stuttgart (Schweitzerbart'sche Verlagsbuchhandlung).

- \& TRESKATIS, C. (1989): Hydrogeologie der Stauchmoräne Hochwald-Balberger Wald im Xantener Lobus. - Natur am Niederrhein (N. F.) 4 (1): S. 1 - 10, 6 Abb., 1 Tab.; Krefeld.

MiNISTER FÜR UMWELT, RAUMORDNUNG UND LANDWIRTSCHAFT (MURL) (Herausgeber) (1989): Klima-Atlas von Nordrhein-Westfalen. - 65 S., 22 Abb., 17 Tab., 51 Karten; Düsseldorf.

Treskatis, C. (1990): Hydrogeologische Standortuntersuchungen bei der Verlagerung der Trinkwassergewinnung aus der Niederterrasse des Rheins in die Stauchmoräne des Staatsforstes Marienbaum-Xanten. Mitt. Ing.- u. Hydrogeologie 36: S. 177 - 207, 11 Abb.; Aachen (RWTH).

Manuskript eingegangen am 20.7.1992 